\title{
A Review of Design Criteria for Low Voltage DC Distribution Stability
}

\author{
Kyle Smith \\ University of Strathclyde, U.K. \\ Kyle.Smith@strath.ac.uk
}

\author{
Stuart Galloway \\ University of Strathclyde, U.K. \\ Stuart.Galloway@strath.ac.uk
}

\author{
Graeme Burt \\ University of Strathclyde, U.K. \\ Graeme.Burt@strath.ac.uk
}

\begin{abstract}
The performance advantages for Low Voltage Direct Current electrical distribution is becoming clearer however, the commercial opportunities, design processes and standardisation is currently lacking. This paper presents an overview of the current development status of LVDC distribution and reviews the modelling and stability criteria available to designers of DC distribution systems for land, aerospace and marine power system applications.

Index Terms-D.C. distribution, microgrids, modelling, stability.
\end{abstract}

\section{INTRODUCTION}

In the context of the energy trilemma (the provision of sustainable, affordable, reliable energy) the application of Low Voltage Direct Current (LVDC) distribution offers several advantages over the incumbent $\mathrm{AC}$ distribution system. In particular, LVDC distribution can increase the power transfer capability of existing power network cable assets and simplify the integration of native DC distributed generators and modern power electronic based loads to the network [1]. Furthermore, it offers the opportunity to interconnect ('mesh') DC based microgrids without the added complexities of frequency and phase synchronisation that AC systems possess [2].

Much of the work on the stability of DC distribution systems has focused on aerospace and marine power systems as opposed to land-based power systems [3],[4]. However, the increase in native DC generation such as solar PV, battery storage and the growing volume of power electronic loads makes a compelling case for the wider deployment of DC distribution on the low voltage network and therefore an improved understanding of system stability is required for these land-based applications.

The IEC has defined the upper limit of LVDC at $1500 \mathrm{~V}$ however, distribution standards are currently being established for specific applications such as $24 / 48 \mathrm{~V}$ lighting systems and $380 \mathrm{~V}$ domestic and data centre distribution [5]. Research trials and niche commercial deployments of LVDC systems have occurred predominantly on the $400 \mathrm{~V}$ network with the intent to alleviate new network constraints and reduce power converter efficiency losses [6]. Although in its nascent stage of development in land based grids, LVDC distribution offers an improved platform in which smarter, low-carbon power networks can evolve.
In aerospace and marine power applications the use of DC distribution or Power Electronic Distribution Systems (PEDSs) is known to offer improved load regulation, transient performance and increased fault tolerance [7]. Despite almost 40 years of ongoing work in the field of DC power system stability, it continues to remain an area of active research with the focus on identifying stability design criteria that yield optimised power systems based on cost, physical size and efficiency [4]. The literature highlights system instabilities associated with tightly regulated power converters that act as Constant Power Loads (CPLs) which supply steady power to the load despite fluctuations on the DC bus input voltage. Under these conditions, the converters act as a negative incremental impedance which can cause system instabilities if left unmanaged [4], [3], [8], [9].

The presence of multiple converters on a shared DC network generally requires an examination of the input and output impedance for each converter and the application of a stability criterion to ensure overall system stability at the design stage. A number of stability criteria exist that offer varying degrees of accuracy and design conservativeness, this paper draws together appropriate stability criteria for five different arrangements of PEDSs[9].

This paper begins with a review of the analytical stability methods used in classical control theory to demonstrate their application in determining DC system stability. Five different DC distribution network topologies are then presented which could be applied to land, aero and marine applications. In each case suitable stability design criteria for the specific application are provided to aid system designers. Finally a review of the methods that can be applied to improve system stability is offered.

\section{ClassificAtion OF StABILITY AND Methods OF ANALYSIS}

The stability of AC power systems is a widely studied subject which involves the analysis of voltage, frequency and rotor angle differences in synchronous machines for both transient and steady state disturbances [10]. In the case of DC power systems, analysis of system stability is focused on maintaining a constant DC bus voltage under both normal operating conditions, where small perturbations occur regularly, and in transient fault conditions where a larger change in load or generation occurs. A system is considered to be stable if it can 
return to its normal operating condition after being subjected to any of these disturbances.

In designing DC distribution systems, much of the academic literature has focused on system stability during steady state operating conditions which involves expected changes in load or generator output. During these disturbances, the system can be linearized and analyzed for stability at fixed operating points. This approach is known as small signal stability analysis and is the focus of this review for DC distribution system design [10].

A small signal stability assessment can be conducted for a DC distribution system using any of the methods presented in Fig.1. The stability of the system depends on the location of the system transfer function poles on the complex $s$-plane but further tools can be employed to assess the margin of stability and the movement of system poles under varying operating conditions.

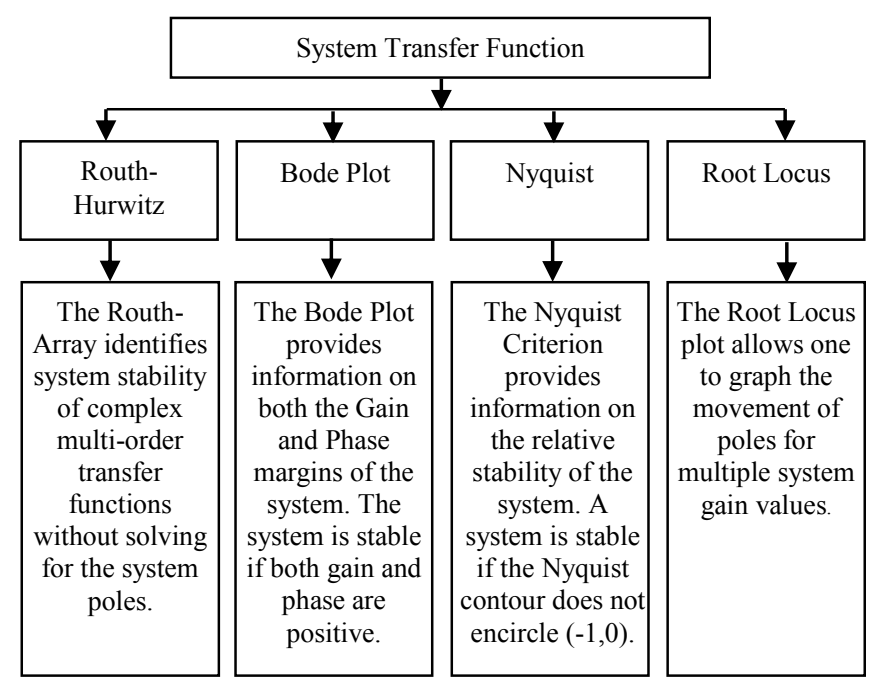

Fig.1. Classical control theory methods of assessing system stability.

In the literature, the majority of DC distribution system stability assessments relate to networks of converters on a single bus which can take the form of a single source and load; single source and multiple loads; multiple parallel sources and loads. The stability of these systems is generally based on the minor loop gain which is the ratio of input and output impedance of the connected converters. There exist multiple criteria in which to evaluate system stability using the minor loop gain. Each evolution of this impedance based stability criteria offers an improved (less conservative) forbidden region on the complex $s$-plane in which the Nyquist contour should avoid in order to ensure sufficient system stability. These stability criteria boundaries are presented in Fig. 2 according to Riccobonno's assessment [9]. It appears that less research has been conducted in the area of interconnected DC microgrids that are geographically dispersed. These systems require the consideration of network cable impedance on overall system stability [11], [12].
Appropriate stability criteria for each of these DC distribution system configurations are considered in the following section.

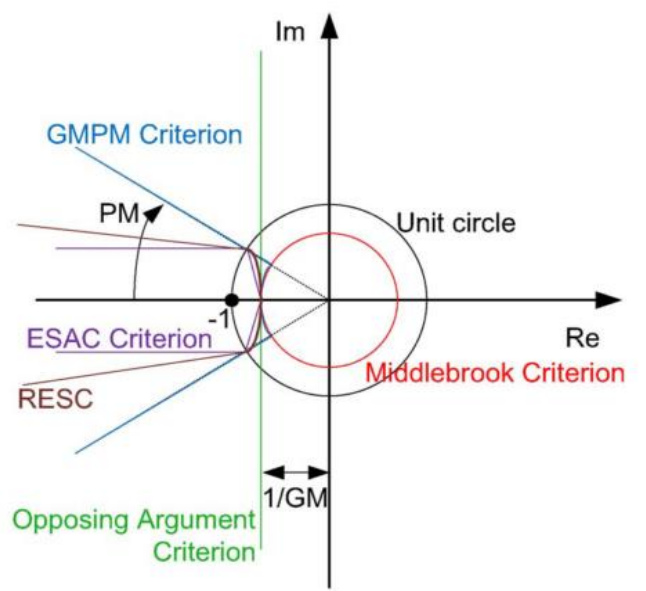

Fig.2. Stability criteria boundaries on the complex $s$-plane demonstrate varying sizes of forbidden areas on the Left Hand Plane (LHP) depending on the chosen stability criterion [9].

\section{D.C. POWER SYSTEM TOPOLOGIES}

This section presents five different DC power system topologies with suitable stability design methodologies for each system. In each case it is assumed that the source converter provides a common DC bus level voltage that could be fed by the AC grid, an AC based distributed generator or a DC based distributed generator.

\section{A. Single source, single load}

The Middlebrook Criterion was developed in 1976 to investigate the stability and dynamic response of a simple source and load sub-system with the addition of an input filter [9]. This method has become the basis for impedance based stability criteria by representing the minor loop gain as the ratio between the modulus of the source converter's output impedance and the load converter input impedance. The stability condition is sufficient if the impedance ratio is less than 1:

$$
\left|Z_{\text {out }}\right|<<\left|Z_{\text {in }}\right| \text { or } T_{M L G}=\left|Z_{\text {out }} / Z_{\text {in }}\right|<<1
$$

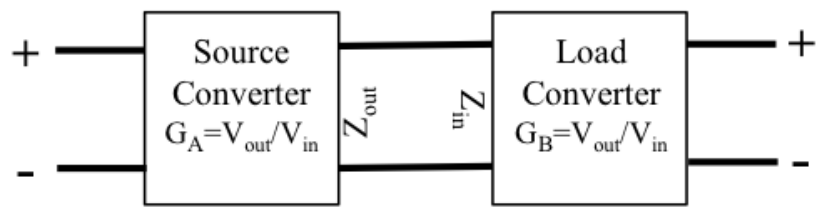

Fig. 3. Single source converter interconnected to a single load converter [9].

This DC system configuration contains a single source converter, which could be fed by a native DC generator or from a wider AC network; this source converter is connected to a single load converter. Both sub-systems are individually stable 
according to their designed operating conditions but when interconnected, the output impedance of the source converter should be greater than the input impedance of the load converter to ensure sufficient system stability.

The Middlebrook Criterion is the most widely used stability criterion due to its ease of application and versatility however, this method is the most conservative stability criterion which does not optimize for component sizing and costs. Ricobonno highlights that although the Middlebrook Criterion is a conservative method, it offers more than system stability, it provides unchanged system dynamics which, for some applications, may be a design requirement [9].

\section{B. Single source, multiple loads}

The next stage in DC network complexity is to consider a system with a single source converter connected to multiple load converters, as depicted in Fig.4. Applications for this system might include a DC electric vehicle charging network, where one centralized converter feeds multiple electric vehicles. Alternatively, a building-level DC lighting or computer distribution system may take this form.

In this case the Middlebrook criterion may be used to design for system stability, however, it is likely that the passive system filter components will be undesirably large and therefore a more refined stability criterion is required to optimize the design for a more complex DC network [9].

The Opposing Argument Criterion (OAC) offers a suitable method to consider the stability of systems with multiple loads connected to a single source. The application of this criterion involves the combined, parallel load impedance of all connected loads [9]. For the system in Fig.4, this creates a minor loop gain that is composed of the output impedance of the source converter $Z_{o}$ and the individual load converter impedances $Z_{\text {in }}$ :

$$
T_{M L G}=\frac{Z_{o}}{Z_{i}}=\frac{Z_{o}}{Z_{\text {in_A }}}+\frac{Z_{o}}{Z_{\text {in_B }}}+\frac{Z_{o}}{Z_{\text {in_C }}} \cdots
$$

According to [9] the forbidden region on the polar plot for a system with a single source and multiple load converters, using the $\mathrm{OAC}$ is defined as:

$$
R e\left(\frac{Z_{o}}{Z_{i n, k}}\right) \leq \frac{1}{G M} \frac{P_{i n, k}}{P_{o}}
$$

Where $k$ represents the input impedance and power for each load converter, this generates a minor loop gain forbidden region for each converter that shifts the vertical line on Fig.2 closer to the $y$-axis as a proportion of each converter's power demand. This is illustrated in the Fig. 2 boundary criteria.

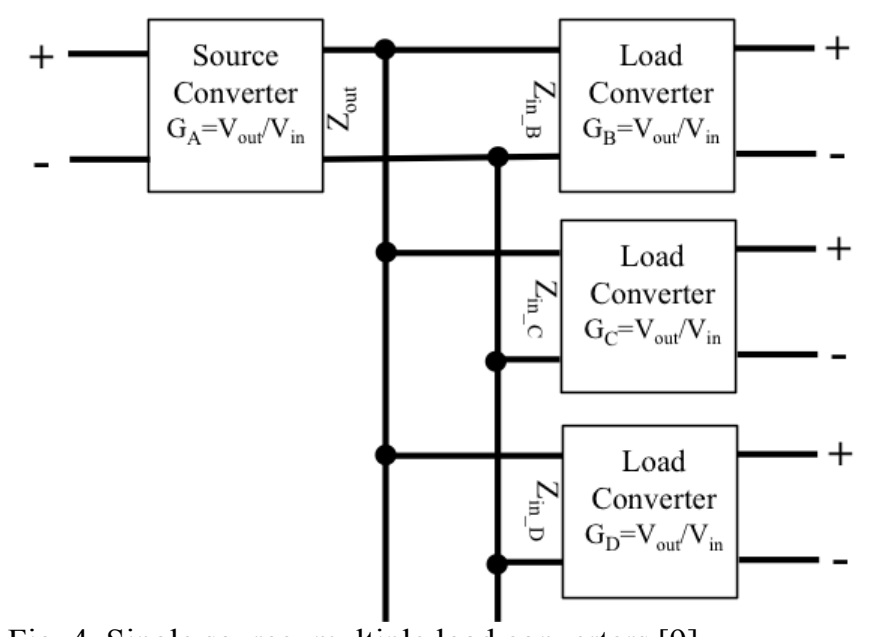

Fig. 4. Single source, multiple load converters [9].

An alternative to the OAC is the Energy Source Analysis Consortium Criterion (ESAC) that was developed by Purdue University [13]. The ESAC criterion further reduces the area of the forbidden region on the Nyquist plot and therefore the associated design conservativeness. According to Riccobono, the ESAC criterion suffers less from component grouping but it is computationally heavy and is not conducive to an easy design solution, furthermore it cannot be used to determine system stability with bi-directional power flows [9].

\section{Paralleled sources, multiple loads}

By adding additional sources in parallel to the previous network example in Fig.4, the system designer requires an alternative, more comprehensive design criterion to ensure sufficient system stability that can take into consideration the combined impedances of the sources and loads

A DC system topology of this type of configuration could take the form of a solar PV and battery storage system each linked to a common DC bus, with a separate source converter, and interconnected to multiple DC/DC load converters controlling the voltage to loads operating at different levels.

Riccobono and Santi have proposed the Passivity Based Stability Criteria (PBSC) for more complex Power Electronic Distribution Systems [14]. This method does not follow the conventional minor loop gain based stability criteria, it instead pursues a 'passivity' solution for the main DC bus. This criterion assumes that a DC bus has a series of source converters connected to a series of load converters that are refined into a single port linear electrical network, as shown in Fig 5.

The common DC bus impedance is determined through the parallel combination of all the converters' input/output impedances [9]:

$$
Z_{\text {bus }}=Z_{s} / / Z_{\text {in }}=Z_{1} / / \ldots / / Z_{n} / / Z_{n+1} / / \ldots / / Z_{n+m}
$$


This method demonstrates that if 'passivity' of the main DC bus can be assured then overall system stability is achieved [14]. A DC network, on a common bus, is considered passive if it can only absorb energy. This can be verified if the impedance transfer function for the system $Z(s)$ has no right half plane (RHP) poles and if $\mathrm{Z}(\mathrm{jw})$ has a Nyquist contour which lies wholly in the closed RHP [14].

The PBSC appears to offer an improved and versatile approach to DC distribution system stability as it can be applied to any combination of source and load converters on a common DC bus, it reduces the design conservativeness compared to the Middlebrook criterion and its derivatives, while also being applicable to systems with bi-directional power flow [14].

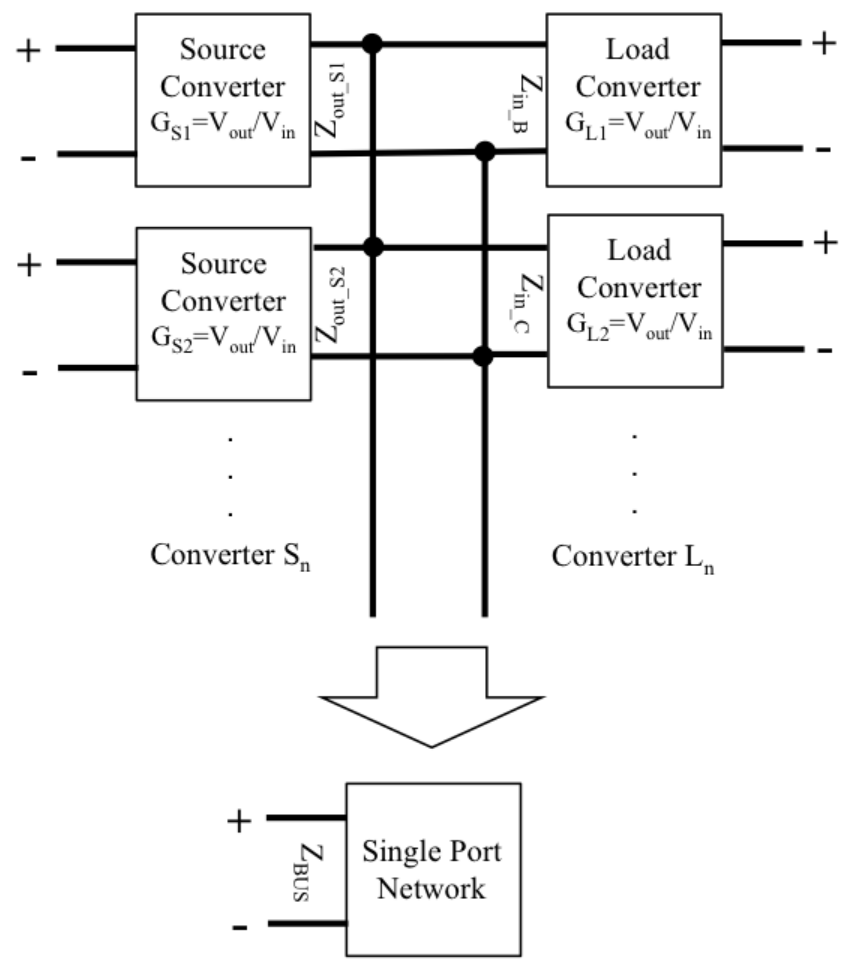

Fig.5. Multiple sources and load converters connected to common DC bus and refined into a single port network for determination of overall system impedance and passivity [9].

\section{Bi-directional sources and loads}

The analysis of DC systems presented previously has assumed a fixed power flow direction but this is not always the case. In networks where a battery energy storage system or super-capacitor exist, the source and load converters can change operating roles and therefore the input and output impedance ratio become inverted, creating a minor loop gain that is less than one. In this scenario, the Middlebrook Criterion fails and an alternative stability criterion should be applied.

The PBSC is suitable for the stability analysis of bi-directional power flow systems [14]. However, Xia and Ayanaar [15] contend that an impedance based approach to the stability of bi-directional power flows can be applied but instead of considering the input and output impedance of the sub-systems, the designer should work with the voltage source $\left(Z_{v}(s)\right)$ and current source $\left(Z_{i}(s)\right)$ impedance of the connected devices. If $Z_{v}(s) / Z_{i}(s)$ conforms to the Nyquist stability criterion then the system is considered stable.

\section{E. Interconnected sources, multiple loads}

Thus far the stability criteria presented in this paper has been concerned with ensuring stability of a single bus DC system. However, as LVDC distribution systems advance it will become necessary to interconnect multiple DC buses with distribution cables where the impedance of these cables must be considered in the overall system stability analysis.

Anand and Fernandes address this DC distribution configuration in [11] by grouping individual DC buses that contain at least one source and load converter into representative nodes. These nodes are then interconnected by distribution cables as illustrated in Fig.6.

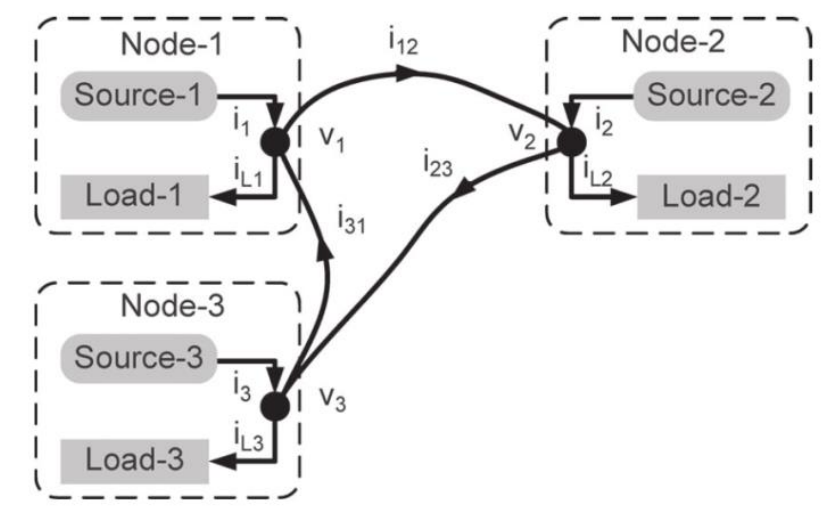

Fig.6. DC Distribution for a 3-node microgrid from [11] where $\mathrm{V}$ represents the voltage at each node and $\mathrm{i}_{\mathrm{ab}}$ the current between nodes $i_{\text {Ln }}$ the current within each node.

The resulting stability analysis approach adds an additional layer of complexity by incorporating droop control of the source converters into their stability assessment. Their approach to system stability analysis involves the formulation of small signal models for system components and first order linear differential equations to describe system behavior under specific operating conditions [11].

Anand and Fernandes conclude that the system stability is improved when the interconnecting cable resistance increases and inductance decreases. This DC distribution topology (Fig.6) may be applied in novel, interconnected microgrids for land based systems or on marine vessels where multiple generating sources are connected over longer distances. However, increasing cable resistance and inductance must be optimised against the system energy efficiency requirements.

\section{STABILITY SOLUTIONS}

The previous section presented an overview of stability methods and criteria that can be applied to specific DC distribution applications. However, understanding whether the 
system is stable is just one half of the design challenge. The other half involves the application of passive system components or active converter control in order to rectify any instability that is identified during the analysis.

The process of stabilising a DC distribution system with CPLs can be achieved using either passive damping methods or active stabilisation methods [16]. Passive solutions include the introduction of resisters, filters and storage units such as batteries or super-capacitors. Despite the benefits they bring passive approaches still introduce energy losses to the system and increase overall complexity. Whereas active damping solutions such as linear and boundary controllers can offer oscillation damping through virtual resistances controlled by proportional and derivative gains [17]. . The active damping approach has a limited impact on the energy efficiency of the network and offers space and weight savings to the design engineer but solutions such as linear controllers may not guarantee global system stability [16], [17].

The controlled shedding of load connected to a point converter can limit the amplitude of voltage oscillations associated with a CPL. However, oscillations will not be fully eliminated and will still require additional resistance to be added with the resulting impact on system efficiency [17]. Load shedding can normally only be applied to non-critical loads and may therefore only be suitable in limited applications.

A further solution is the use of super capacitors to eliminate oscillations associated with CPLs but due to their low cell voltage these capacitors require power electronics to interface with a higher DC microgird distribution voltage of a 300 $1500 \mathrm{~V}$ [8],[17]. The presence of a converter in this case would to a certain extent negate the stabilising effect of the super capacitor.

Passive solutions are difficult to implement from the perspective of a systems integrator as most components in the system will be 'off the shelf' products and therefore changes to the converter hardware may not be practical [4]. However, the designer will have control over the bus impedance and the interconnecting cables between buses which can be tailored according to the stability requirements. Therefore it is likely to prove more practical to manage converter control systems in order to ensure system stability under all operating conditions.

\section{FURTHER WORK}

Much of the work on the stability of DC distribution systems relates to the CPL effect of compact DC networks in marine and aerospace applications. The research in this field has been led by Hodge and Flower in the U.K. [3], [18] and by Glover and Sudoff in the US for almost 20 years where 'all electric propulsion' for naval ships has been pursued. More recently, Ricobonno and Santi have offered an improved stability criterion for DC systems with complex loading regimes [14]. Future work may consider an integrated approach to stability and protection system design to optimise the passive component sizes for inductive, resistive and capacitive values that will enable the effective operation of the protection system while ensuring system stability.

In the area of LVDC distribution for land-based systems, Lappeenranta Technical University of Finland have made significant progress towards the technical viability of point to point DC distribution however, little information exists on any stability challenges that were encountered during this installation [19], [20]. Kwasinski and Onwuchenkwa [17] as well as Chen and $\mathrm{Xu}$ [12] have made contributions towards understanding and designing for system stability in DC microgrids. The development of both point-to-point distribution and DC microgirds can still benefit from increased operational testing that will validate stability design criteria and help to quantify the benefits that DC distribution could provide over AC systems. Furthermore, the system stability analysis could be extended to include the wider $\mathrm{AC}$ grid to assess the impact that a DC distribution system may have on the existing AC network.

\section{CONCLUSIONS}

This paper has introduced the CPL effect for DC distribution systems and the associated instability caused by the interconnection of multiple converter sub-systems onto a common DC bus. Individually, each power electronic converter is stable under its designed operating conditions but collective interaction of these converters can cause system instability

Five DC distribution topologies were presented and possible stability criteria were examined for each topology. The Middlebrook stability criterion was highlighted as the system designer's preferred criteria however, where complex loading conditions exist, the Passivity Based Stability Criterion offers many advantages over other stability criteria.

Finally, passive and active stability solutions were discussed in order to maintain system stability. It was noted that active stabilsation solutions offer the most energy efficient and versatile option for ensuring DC system stability, this is especially true in applications where weight and physical size are considered design constraints. This work will go onto investigate practical applications for land-based LVDC distribution with a focus on the design requirements that will evaluate options for increased deployment.

\section{ACKNOWLEDGEMENTS}

This work has been supported through the EPSRC Centre for Doctoral Training in Future Power Networks and Smart Grids (EP/L015471/1) with industry support from Rolls-Royce plc. 


\section{REFERENCES}

[1] D. Antoniou et al., "DC utilization of existing LVAC distribution cables," 2013 IEEE Electr. Insul. Conf. EIC 2013, pp. 518-522, 2013.

[2] S. Backhaus et al., "Los Alamos National Laboratory DC Microgrids Scoping Study - Estimate of Technical and Economic Benefits."

[3] C. G. Hodge and J. O. Flower, "DC power system stability," Electr. Sh. Technol. Symp. 2009. ESTS 2009, no. April, pp. 433-439, 2009.

[4] S. D. Sudoff and S. F. Glover, "Modeling Techniques, Stability Analysis, and Design Criteria for DC Power Systems with Experimental Verification," J. Aerosp., vol. 107, 1998.

[5] IET, Code of Practice for Low \& Extra Low Voltage Direct Current Power Distribution in Buildings. 2015.

[6] P. Nuutinen et al., "Research Site for Low-Voltage Direct Current Distribution in a Utility Network Structure, Functions, and Operation," Smart Grid, IEEE Trans., vol. PP, no. 5, p. 1, 2014.

[7] S. D. D. Sudhoff et al., "Admittance space stability analysis of power electronic systems," Aerosp. Electron. Syst. IEEE Trans., vol. 36, no. 3, pp. 965 973, 2000.

[8] A. Kwasinski and P. T. Krein, "Stabilization of constant power loads in Dc-Dc converters using passivity-based control," INTELEC, Int. Telecommun. Energy Conf., pp. 867-874, 2007.

[9] A. Riccobono and E. Santi, "Comprehensive review of stability criteria for DC distribution systems," 2012 IEEE Energy Convers. Congr. Expo., pp. 3917-3925, 2012.

[10] P. Kundur, Power System Stability \& Control. McGraw-Hill, 1994.

[11] S. Anand and B. G. Fernandes, "Reduced-order model and stability analysis of low-voltage dc microgrid," IEEE Trans. Ind. Electron., vol. 60, no. 11, pp. 5040-5049, 2013.

[12] D. Chen et al., "DC network stability and dynamic analysis using virtual impedance method," IECON 2012 - 38th Annu. Conf. IEEE Ind. Electron. Soc., pp. 5625-5630, 2012.

[13] P. University, "DC Stability Toolbox 3.0.” [Online]. Available: https://engineering.purdue.edu/ECE/Research/Areas/ PEDS/dc_stability_toolbox.

[14] A. Riccobono and E. Santi, "A novel Passivity-Based Stability Criterion (PBSC) for switching converter DC distribution systems," Conf. Proc. - IEEE Appl. Power Electron. Conf. Expo. - APEC, pp. 2560-2567, 2012.

[15] Y. Xia and R. Ayyanar, "Influence of bi-directional power flow on impedance and stability of cascaded systems," Proceedings, IECON 2014 - 40th Annu. Conf. IEEE Ind. Electron. Soc., pp. 1542-1548, 2015.

[16] R. Ahmadi, "Dynamic modeling, stability analysis , and controller design for DC distribution systems,"
2013.

[17] A. Kwasinski and C. N. Onwuchekwa, "Dynamic behavior and stabilization of DC microgrids with instantaneous constant-power loads," IEEE Trans. Power Electron., vol. 26, no. 3, pp. 822-834, 2011.

[18] J. O. F. Dsc et al., "Stability and transient-behavioural assessment of power-electronics based dc-distribution systems .," pp. 1-6.

[19] a Mattsson et al., "Implementation of a Modular Customer-End Inverter for a Low Voltage DC Distribution Network," Power Electron. Appl. (EPE'14-ECCE Eur. 2014 16th Eur. Conf., pp. 1-10, 2014.

[20] P. Salonen et al., "Protection scheme for an LVDC distribution system," no. 0891, pp. 1-4, 2009. 